COMMENT. The authors cite only one other child with these symptoms reported in the literature, and 10 adult cases. Patients with seizures and coma of undetermined cause should be tested for hyperthyroidism.

\title{
TREATMENT OF NEONATAL SEIZURES
}

Controversies in treatment of neonatal seizures are addressed by neurologists from Stanford and Yale Universities. Seizures likely to be associated with electrographic seizure activity (eg focal clonic or multifocal clonic seizures) should be treated with antiepileptic drugs (AEDs). Bilateral tonic posturing, unaccompanied by EEG seizure activity, would not be benefited by AEDs. The presence of seizures should be determined electrographically before treatment is begun. For symptomatic seizures, AEDs are administered until clinical seizures, but not necessarily all EEG seizures, are controlled. The nature and severity of the underlying cerebral lesions are the major determinants of outcome. Conventional treatment, usually with phenobarbital, is not very effective; control is obtained in only one-third of patients. The goals are to restore a normal metabolic state, control hypertension and tachycardia, and reduce the increase in glucose utilization. (Hahn JS, Young RSK. Controversies in treatment of neonatal seizures. Pediatr Neurol July/Aug 1993;9:330-332).

COMMENT. Seizures caused by hypoxic-ischemic encephalopathy and intracranial hemorrhage are the most refractory to treatment with AEDs. Seizures due to metabolic abnormalities are usually transitory and require early recognition and specific management. Water intoxication as a potential etiology of seizures in young infants, a previously rare condition, is increasing to epidemic proportions in US inner cities (Keating JP, Dodge PR; and Furth S, Oski FA. AIDC Sept 1993;147:932-3). The maternal motivation for offering water remains uncertain. Improper dilution of formula and feeding large amounts of free water or glucose water, especially at times of diarrhea, are common practices, and occasionally result from improper medical advice. Infants with seizures refractory to AEDs should be tested for hyponatremia.

\section{CARBAMAZEPINE CARDIOTOXICITY}

A 13-month-old boy with complex partial seizures complicating tuberous sclerosis developed a cardiac conduction abnormality and heart block during treatment with carbamazepine in a report from the University of Massachusetts Medical School, Worcester, MA. The CBZ serum level was 6 $\mathrm{mcg} / \mathrm{ml}$ and CBZ-10,11-epoxide level, $1 \mathrm{mcg} / \mathrm{ml}$. After CBZ was discontinued a repeat EKG was normal. The echocardiogram, at first thought to show a small rhabdomyoma, was normal on repeat study 2 years later. (Weig SG, Pollack P. Carbamazepine-induced heart block in a child with tuberous sclerosis and 
cardiac rhabdomyoma: Implications for evaluation and follow-up. Ann Neurol

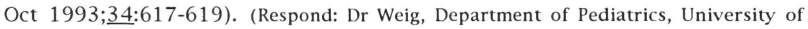
Massachusetts Medical Center, Worcester, MA 01655).

COMMENT. The authors cite one previous report of heart block secondary to erythromycin-induced CBZ toxicity in a 10-year-old boy. This child had no evidence of underlying cardiac disease. Additional cases in adults have been reported. Although CBZ cardiotoxicity is rare, an echocardiogram is recommended in patients with tuberous sclerosis treated with carbamazepine, and cardiac rhythm should be carefully monitored.

\section{ICTAL SPECT IN LOCALIZING EPILEPTOGENESIS}

Ictal single-photon emission computed tomography (SPECT) was used successfully in the localization of an epileptic focus in two children, ages 9 and 7 years, with intractable epilepsy reported from the University of Alabama at Birmingham Epilepsy Center. CT and initial MRIs were normal, and repeated EEG-video monitoring studies failed to localize the seizures. Resection of the abnormal areas shown by SPECT as hyperperfusion in the frontal lobes revealed a cortical dysplasia. The children had no neurological deficits and remained seizure free 1 year after surgery. (Kuzniecky R et al. Ictal singlephoton emission computed tomography demonstrates localized epileptogenesis in cortical dysplasia. Ann Neurol Oct 1993; $\underline{34: 627-631) . ~(R e s p o n d: ~ D r ~ K u z n i e c k y, ~}$ Department of Neurology, UAB Station, Birmingham, AL 35294).

COMMENT. Ictal SPECT performed exactly at the onset of a clinical seizure precisely localized the primary epileptogenic area in the cortical dysplasia and permitted a discrete resection of the focus without neurologic deficit.

\section{TEMPORAL LOBE TUMOR AND LANDAU-KLEFFNER SYNDROME}

A 7-year-old boy with a left temporal lobe tumor and Landau-Kleffner syndrome (acquired aphasia and epilepsy) who improved dramatically after surgery is reported from the New York Hospital-Cornell University Medical Center. A verbal auditory agnosia with mutism had evolved over a 3 month period after onset of refractory partial complex seizures at 5 years. EEG showed left temporal and right occipital spike and $\mathrm{S} / \mathrm{W}$ discharges. Language comprehension was absent on the Peabody Picture Vocabulary test and Token test. Following removal of a grade 1 astocytoma demonstrated on MRI, seizures stopped, language returned, and after 1 year speech and comprehension were almost normal for age. (Nass R et al. Landau-Kleffner syndrome: Temporal lobe tumor resection results in good outcome. Pediatr Neurol July/Aug 1993; $\underline{9}: 303-5)$. (Respond: Dr Nass, New York Univ Med Ctr, 400 E 34th St RR 212, New York, NY 10021). 